\title{
Gauge Covariance and the Chiral Condenate in QED3
}

\author{
A. Bashir ${ }^{1,2}$ and A. Raya ${ }^{1}$ \\ ${ }^{1}$ Instituto de Física y Matemáticas, Universidad Michoacana de San Nicolás de Hidalgo, \\ Apartado Postal 2-82, Morelia, Michoacán 58040, México and \\ ${ }^{2}$ IPPP, Durham University, Durham DH1 3LE, United Kingdom. \\ (Received on 13 September, 2006)
}

\begin{abstract}
The ambiguities associated with the lack of gauge invariance in the non-perturbative truncations of Schwinger-Dyson equations (SDEs) are a challenging problem which has not yet been resolved in a decisive fashion. Pursuing this aim, we study dynamical chiral symmetry breaking in quantum electrodynamics in three space-time dimensions (QED3). We investigate the gauge dependence of the chiral condensate both in the quenched and the unquenched versions of the theory and emphasize the importance of taking into account the gauge covariance properties of the fermion propagator as dictated by its Landau-Khalatnikov-Fradkin transformation (LKFT). We present numerical solutions of the SDE of the fermion propagator which respect Ward-Green-Takahashi identities (WGTI) and LKFT simultaneously. As a striking consequence, we obtain a practically gauge independent chiral condensate.
\end{abstract}

Keywords: Schwinger-Dyson equations; Dynamical Chiral Symmetry Breaking; Landau-Khalatnikov-Fradkin transformations

\section{INTRODUCTION}

Gauge theories of fundamental interactions have been highly successful in collating experimental results in the perturbative regime. However, not all interesting phenomena can be understood in this approximation scheme. Confinement of quarks and gluons and the origin of hadronic masses are two examples. It is well known that on the distance scale of the order of hadron size, quarks behave as though their mass were $300 \mathrm{MeV}$, much larger than their vanishingly small current mass. In the context of covariant gauges, this effect may be attributed to an interplay of the behaviour of gluon and ghost propagators in the infrared, [1]. These studies through Schwinger-Dyson equations (SDEs) have only been carried out in the Landau gauge. It is well known that if care is not taken in their truncation, solutions can be gauge dependent.

Let alone the complicated battle ground of non-Abelian theories, the ambiguities associated with the lack of gauge invariance in the non-perturbative truncations of SDEs in Abelian theories such as QED are also a challenging problem. Among others isuues, it has ultraviolet divergences. Consequently, a neater testing ground for the validity of various truncations is provided by QED3, which is super-renormalizable. However, a recent study, [2], has revealed that the problem of gauge invariance persists even in this seemingly simple scenario.

In addition to being a toy model for SDEs studies, QED3 is an attractive theory in its own right. In condensed matter physics, for example, it is used in the context of high- $T_{c}$ superconductors [3], studies which have received a boost from new experiments [4], and in the recently explored unconventional Quantum Hall Effect in Graphene [5]. Additionally, in the realm of dynamical generation of fundamental fermion masses, numerical findings on the lattice, results obtained by employing SDEs, [6], and alternative methods, [7], have yet to arrive at a final consensus, and continue to provide a popular battle ground.

The problem of gauge invariance in QED3 can be traced back to not employing (or doing so incorrectly) the gauge identities such as the Ward-Green-Takahashi identi- ties (WGTI) [8], the Nielsen identities [9] and the LandauKhalatnikov-Fradkin transformations (LKFT) [10]. Here we address this issue in the light of the LKFT, following and extending the analysis of Ref. [11]. These transformations describe the specific manner in which Green functions transform under a variation of gauge. These are non-perturbative in nature, and thus are expected to be very helpful in studies of phenomena realized only non perturbatively, such as dynamical chiral symmetry breaking. Initial steps were taken in [12] to apply LKFT directly to the dynamically generated mass function, whereas the complete numerical implementation of LKFTs on solutions to SDEs in various truncation schemes was performed in [11] to study the gauge dependence of the chiral condensate in QED3. In this work we extend the analysis to a wider range of values of the gauge parameter.

The traditional way to study gauge dependence is to make an ansatz for the fermion-boson vertex which should preserve the key features of gauge theories, namely, it should satisfy the WGTI, it should transform gauge covariantly in the way dictated by its LKFT, it should ensure that the fermion propagator obtained as a solution of the corresponding SDE transforms according to its LKFT, it should be free from (tachionic) kinematic singularities, it should possess the same CPT transformation properties as the bare vertex and it should reduce to its Feynman expansion in the weak coupling regime. Some proposals for the fermion-boson vertex are well known, [1320]. Significant progress has been made over the years to construct reliable truncation schemes to incorporate these features (see [21] for a brief review). Since a priori there is no way to determine whether an ansatz performs better or worse, the following methodology is useful to test the consistency of the truncation scheme : One solves the SDE for the fermion propagator in various gauges changing the value of the gauge parameter in small steps away from the Landau gauge. One then measures the improvement when the gauge dependence of physical observables of interest is reduced as compared to previously known truncations. This method does not guarantee that LKFT for the fermion propagator will be satisfied gauge by gauge. In Ref. [11] we suggested an alternative ap- 
proach to achieve the same objective. We propose to start from the solution for the fermion propagator in the Landau gauge and simply perform an LKFT to find the result in any other gauge. It is important to note that LKFT does not fix the fermion propagator in the starting gauge, e.g., the Landau gauge. If one starts from a flawed starting solution, one does not expect to arrive at a trustable answer in an arbitrary gauge through the use of corresponding LKFT. Therefore, it is important to establish the validity of a vertex ansatz in the starting gauge. Once it has been achieved and owing to the fact that LKFT preserve WGTI, the advantage of this proposal is to conserve both the WGTI and LKFT as we move from one value of the gauge parameter to another. In this contribution, we present results coming from the computation of the chiral condensate as a function of gauge both for the quenched and the unquenched cases and find the results to be practically gauge independent.

We have organized this work as follows : In section II, we introduce the general features of the phenomenon of dynamical mass generation in QED3 within the context of SDEs in the Rainbow approximation. In section III, we present the LKFT for the fermion propagator and the strategy for its implementation in SDEs studies. In Sect. IV we review further truncation schemes in the light of the LKFT. Finally, we discuss our results and draw our conclusions in section V.

\section{SCHWINGER-DYSON EQUATIONS AND DYNAMICAL CHIRAL SYMMETRY BREAKING}

We know that perturbation theory in QED does not generate fermion masses dynamically. Non-perturbative methods have to be employed and SDEs are the natural tool for calculation in continuum. The first three equations of the infinite tower of SDEs in QED are depicted in Fig. 1. These correspond to the fermion propagator, the photon propagator and the fermion-boson vertex, respectively. The fermion propagator is coupled to the photon propagator and the fermion-boson vertex, which, through their own SDE, are coupled to the rest of the infinite tower containing higher point Green functions.

The SDE for the fermion propagator in QED3 is :

$$
\begin{aligned}
S^{-1}(p ; \xi) & =S_{0}^{-1}(p) \\
& +e^{2} \int \frac{d^{3} k}{(2 \pi)^{3}} \Gamma_{v}(k, p ; \xi) S(k ; \xi) \gamma_{\mu} \Delta_{\mu v}(q),
\end{aligned}
$$

where $q=k-p$ and $e^{2}$ is the dimensionful coupling of QED3. In this expression $\Delta_{\mu v}(q)$ is the photon propagator and $\Gamma_{\mu}(k, p ; \xi)$ the full fermion-boson vertex. The most general form of the (Ecuclidean) fermion propagator is

$$
S(p ; \xi)=\frac{F(p ; \xi)}{i \not p-\mathcal{M}(p ; \xi)}
$$

where $F$ is referred to as the fermion wavefunction renormalization and $\mathcal{M}$ is the mass function. The photon propagator in its most general form can be written as

$$
\begin{aligned}
\Delta_{\mu v}(q) & =\frac{G\left(q^{2}\right)}{q^{2}}\left(\delta_{\mu v}-\frac{q_{\mu} q_{v}}{q^{2}}\right)+\xi \frac{q_{\mu} q_{v}}{q^{4}} \\
& \equiv \Delta_{\mu v}^{T}(q)+\xi \frac{q_{\mu} q_{v}}{q^{4}}
\end{aligned}
$$

where $\mathcal{G}$ is the photon wavefunction renormalization, which is gauge invariant, and $\xi$ is the usual covariant gauge parameter. Finally, the fermion-boson vertex can be written as $\Gamma^{\mu}(k, p ; \xi)=\sum_{i=1}^{12} v_{i}(k, p ; \xi) V_{i}^{\mu}$, where

$$
\begin{gathered}
V_{1}^{\mu}=\gamma^{\mu}, \quad V_{2}^{\mu}=k^{\mu}, \quad V_{3}^{\mu}=p^{\mu}, \\
V_{4}^{\mu}=\not k \gamma^{\mu}, \quad V_{5}^{\mu}=\not k k^{\mu}, \quad V_{6}^{\mu}=\not k p^{\mu}, \\
V_{7}^{\mu}=\not p \gamma^{\mu}, \quad V_{8}^{\mu}=\not p k^{\mu}, \quad V_{9}^{\mu}=\not p p^{\mu}, \\
V_{10}^{\mu}=\not k p \gamma^{\mu}, \quad V_{11}^{\mu}=\not k p k^{\mu}, \quad V_{12}^{\mu}=\not k p p p^{\mu} .
\end{gathered}
$$
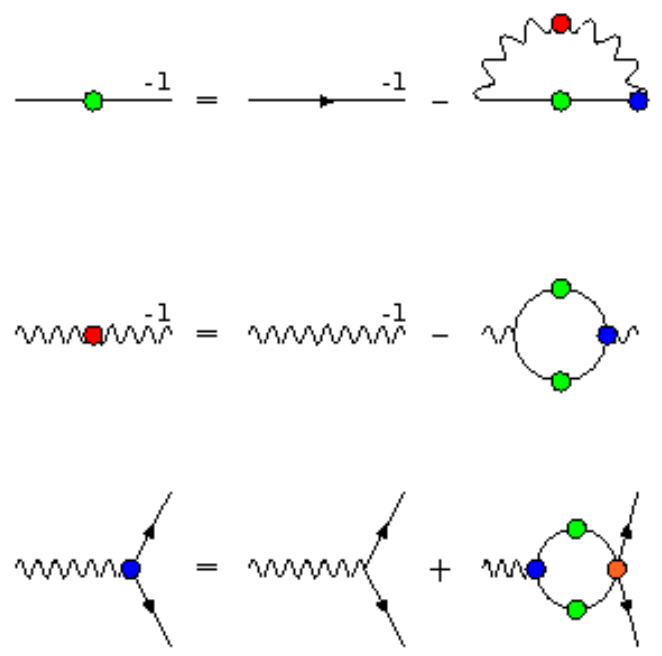

FIG. 1: SDEs for the fermion propagator, photon propagator and fermion-boson vertex.

Expression (1) is a matrix equation which can be converted into system of coupled non-linear scalar integral equations for $\mathcal{M}$ and $F$ after multiplying it, respectively, by 1 and $\not p$ and taking trace. A favorite starting point in the quenched version of QED3, which consists in neglecting fermion loops $(G=1)$, is to choose a suitable ansatz for the fermion-boson vertex, in such a way that the SDE for the fermion propagator can be solved for the unknowns $\mathcal{M}$ and $F$. Possibly the simplest choice for the vertex, which allows a neat understanding of the general features of dynamical chiral symmetry breaking, consists in replacing the fermion-boson vertex by its bare counterpart $[15,22]$. This is the so-called Rainbow approximation. To start with, the bare fermion propagator is considered massless. In this case the unknown functions defining the fermion propagator are found through the following system of equations: 
$\frac{1}{F(p ; \xi)}=1-\frac{\alpha \xi}{4 \pi} \int_{0}^{\infty} d k \frac{k^{2} F(k ; \xi)}{k^{2}+\mathcal{M}^{2}(k ; \xi)}\left[1-\frac{k^{2}+p^{2}}{2 k p} \ln \left|\frac{k+p}{k-p}\right|\right]$

$$
\frac{\mathcal{M}(p ; \xi)}{F(p ; \xi)}=\frac{\alpha(\xi+2)}{\pi p} \int_{0}^{\infty} d k \frac{k F(k ; \xi) \mathcal{M}(k ; \xi)}{k^{2}+\mathcal{M}^{2}(k ; \xi)} \ln \left|\frac{k+p}{k-p}\right|,(5)
$$

where $\alpha=e^{2} / 4 \pi$ as usual. In the Landau gauge $F(p ; 0)=$ 1 and thus we have to solve but one equation for the mass function :

$$
\mathcal{M}(p ; \xi)=\frac{2 \alpha}{\pi p} \int_{0}^{\infty} d k \frac{k \mathcal{M}(k ; \xi)}{k^{2}+\mathcal{M}^{2}(k ; \xi)} \ln \left|\frac{k+p}{k-p}\right| .
$$

In Fig. 2, we show the profile of the mass function, Refs. [15, 22]. $\mathcal{M}(p ; 0)$ is roughly a constant for low momentum and fall as $1 / p^{2}$ as $p \rightarrow \infty$, [22].

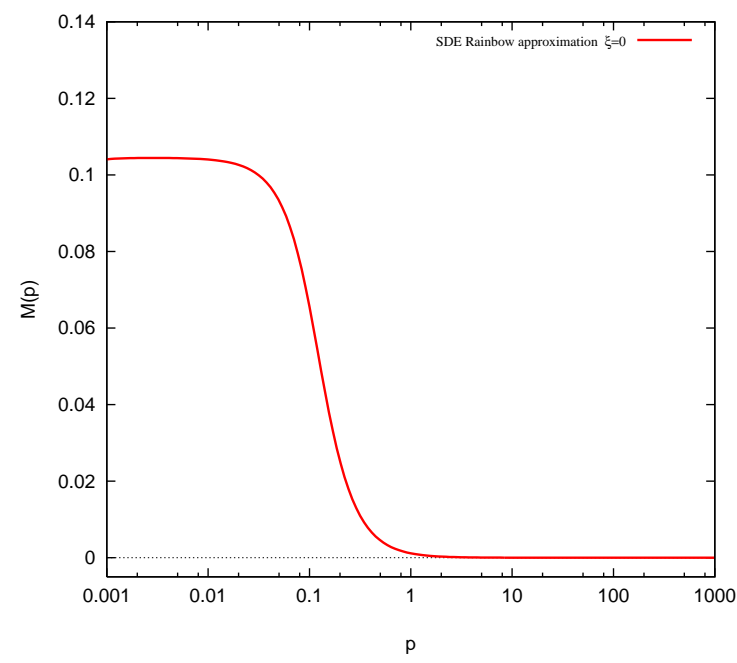

FIG. 2: Mass function from SDE in the Landau gauge in the Rainbow approximation.

From this solution can can compute the chiral condensate $\langle\bar{\psi} \psi\rangle=-\operatorname{Tr} S(x=0, \xi)$. When this exerecise is performed in different gauges, i.e., solving simultaneously the eqs. in (5), one faces the undesirable fact of the condensate being gauge dependent, as shown in Fig. 3. One can expect the source of such gauge dependence the fact that the bare vertex violates the WGTI

$$
i q_{\mu} \Gamma_{\mu}(k, p ; \xi)=S^{-1}(k ; \xi)-S^{-1}(p ; \xi) .
$$

Nevertheless, this identity is satisfied in the Landau gauge (as $F(p ; 0)=1)$ upto a correction connected with the difference $\mathcal{M}(k ; 0)-\mathcal{M}(p ; 0)$ of the mass function. This correction might be expected to be small because $\mathcal{M}(k ; 0) \sim \mathcal{M}(p ; 0)$ for small values of momenta and falls sharply for large momenta. In other gauges, $F(p ; \xi) \neq 1$ and the above argument does not apply. Thus, if one insists on sticking to the Rainbow approximation, only the results in the Laundau gauge can be reliable. However, one could use the LKFT to generate solutions in other gauges [23]. Below we present the corresponding analysis for the chiral condensate.

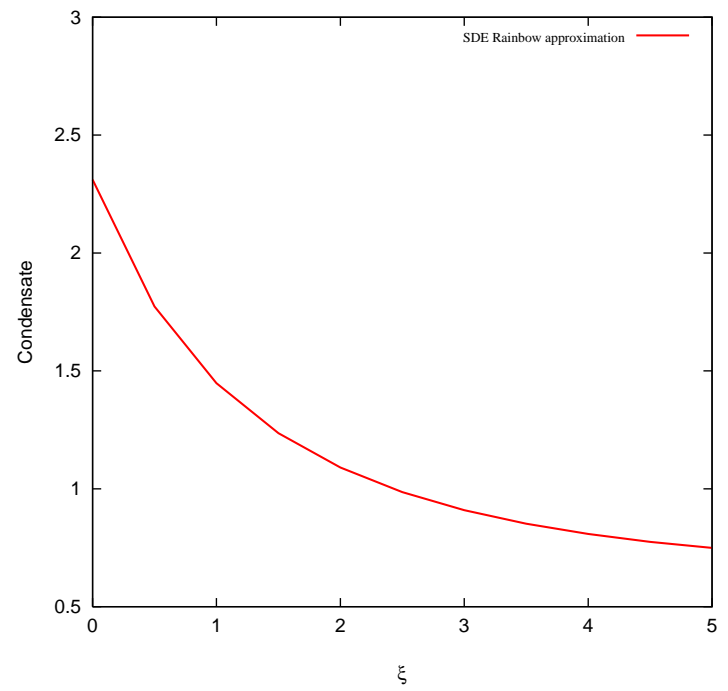

FIG. 3: Gauge dependence of the chiral condensate (in units of $10^{-3} e^{4}$ ) from SDE in the Rainbow approximation.

\section{LANDAU-KHALATNIKOV-FRADKIN TRSNFORMATIONS AND THE FERMION PROPAGATOR}

We start by writing the Euclidean coordinate space fermion propagator in its most general form :

$$
S(x ; \xi) \equiv \not X(x ; \xi)+Y(x ; \xi) .
$$

Expressions in eqs. (2) and (8) are related through Fourier transformations :

$$
\begin{aligned}
& S(p ; \xi)=\int d^{3} x \mathrm{e}^{i p \cdot x} S(x ; \xi), \\
& S(x ; \xi)=\int \frac{d^{3} p}{(2 \pi)^{3}} \mathrm{e}^{-i p \cdot x} S(p ; \xi) .
\end{aligned}
$$

Assuming we know the fermion propagator in Landau gauge in momentum space, $S(p ; 0)$, we have the following expressions in coordinate space

$$
\begin{aligned}
& X(x ; 0)=\frac{-i}{x^{2}} \int \frac{d^{3} k}{(2 \pi)^{3}} \frac{F(k ; 0)}{k^{2}+\mathcal{M}^{2}(k ; 0)} e^{-i k \cdot x} k \cdot x, \\
& Y(x ; 0)=-\int \frac{d^{3} k}{(2 \pi)^{3}} \frac{F(k ; 0) \mathcal{M}(k ; 0)}{k^{2}+\mathcal{M}^{2}(k ; 0)} e^{-i k \cdot x} .
\end{aligned}
$$

The LKFT relating the coordinate space fermion propagator in the Landau gauge to the one in an arbitrary covariant gauge reads

$$
S(x ; \xi)=S(x ; 0) \mathrm{e}^{-i\left[\Delta_{d}(0)-\Delta_{d}(x)\right]},
$$


where

$$
\Delta_{d}(x)=-i \xi e^{2} \int \frac{d^{d} p}{(2 \pi)^{d}} \frac{\mathrm{e}^{-i p \cdot x}}{p^{4}}
$$

$d$ being the number of space-time dimensions. For $d=3$, $\Delta_{3}(0)-\Delta_{3}(x)=-i \alpha \xi / 2$. Therefore,

$$
S(x ; \xi)=S(x ; 0) e^{-a x},
$$

with $a=\alpha \xi / 2$. After applying LKFT to the expressions in eq. (10) and Fourier transforming the results back to momentum space, we get

$$
\begin{gathered}
\frac{F(p ; \xi)}{p^{2}+\mathcal{M}^{2}(p ; \xi)}=\frac{a}{\pi p^{2}} \int_{0}^{\infty} d k k^{2} \frac{F(k ; 0)}{k^{2}+\mathcal{M}^{2}(k ; 0)} \times \\
\left.\frac{1}{\lambda^{-}}+\frac{1}{\lambda^{+}}+\frac{1}{2 k p} \ln \left|\frac{\lambda^{-}}{\lambda^{+}}\right|\right] \\
\frac{F(p ; \xi) \mathcal{M}(p ; \xi)}{p^{2}+\mathcal{M}^{2}(p ; \xi)}=\frac{a}{\pi p} \int_{0}^{\infty} d k k \frac{F(k ; 0) \mathcal{M}(k ; 0)}{k^{2}+\mathcal{M}^{2}(k ; 0)} \times \\
{\left[\frac{1}{\lambda^{-}}-\frac{1}{\lambda^{+}}\right]}
\end{gathered}
$$

where $\lambda^{ \pm}=a^{2}+(k \pm p)^{2}$. Thus knowledge of $S(p ; 0)$ is the input required to obtain the same in an arbitrary covariant gauge.

When we insert the solution for the mass function in the Landau gauge, eq. (6), into eqs. (14) and compute the chiral condensate, we find the striking flat curve shown in Fig. 4 (green-dashed), as compared with the SDE solution (redsolid). Furthermore, with this procedure, we can go as far as we wish in varying the gauge parameter (here upto $\xi=200$ ), a hard nut to crack in SDE studies [15, 22].

A linear fit of the gauge dependence of the condensate reveals that $\langle\bar{\psi} \psi\rangle \simeq 2.30783-0.000113777 \xi$, which suggest that the condensate, scaled by a factor of $10^{-3}$, would vanish for a value of $\xi_{v}>20000$, provided a good numerical accuracy is achieved.

\section{FURTHER TRUNCATIONS}

In order to test the procedure outlined above, we review two further examples of truncation schemes of SDEs.

\section{A. Ball-Chiu Vertex}

The Ball-Chiu vertex (BC-vertex), eq. (3.2) in [13] was constructed to fulfill the WGTI non-perturbatively. In the quenched truncation, the corresponding SDEs become more involved. Inserting the BC-vertex in the SDE, the unknown $F$ and $\mathcal{M}$ are found through the system of equations

$$
\begin{aligned}
\frac{1}{F(p ; \xi)}= & 1+\frac{\alpha}{\pi p^{2}} \int_{0}^{\infty} d k \frac{k^{2} F(k ; \xi)}{k^{2}+\mathcal{M}^{2}(k ; \xi)}\left\{a(k, p)\left[-\xi\left(1-\frac{k^{2}+p^{2}}{2 k p} \ln \left|\frac{k+p}{k-p}\right|\right)\right]\right. \\
& +b(k, p)\left[2\left(k^{2}+p^{2}\right)\left(1-\frac{k^{2}+p^{2}}{2 k p} \ln \left|\frac{k+p}{k-p}\right|\right)-\xi\left(k^{2}+p^{2}-\frac{\left(k^{2}-p^{2}\right)^{2}}{2 k p} \ln \left|\frac{k+p}{k-p}\right|\right)\right] \\
& \left.-c(k, p)\left[2\left(1-\frac{k^{2}+p^{2}}{2 k p} \ln \left|\frac{k+p}{k-p}\right|\right)-\xi\left(1-\frac{k^{2}-p^{2}}{2 k p} \ln \left|\frac{k+p}{k-p}\right|\right)\right]\right\}, \\
\frac{\mathcal{M}(p ; \xi)}{F(p ; \xi)}= & \frac{\alpha}{\pi} \int_{0}^{\infty} d k \frac{k^{2} F(k ; \xi)}{k^{2}+\mathcal{M}(k ; \xi)}\left\{a(k, p) \mathcal{M}(k ; \xi)\left[(2+\xi) \frac{1}{k p} \ln \left|\frac{k+p}{k-p}\right|\right]\right. \\
& +b(k, p) \mathcal{M}(k ; \xi)\left[\frac{2\left(k^{2}+p^{2}\right)}{k p} \ln \left|\frac{k+p}{k-p}\right|+2(\xi-2)\right] \\
& \left.+c(k, p)\left[\frac{(2+\xi) k^{2}+(2-\xi) p^{2}}{2 k p} \ln \left|\frac{k+p}{k-p}\right|+(\xi-2)\right]\right\}
\end{aligned}
$$

where

$$
\begin{aligned}
& a(k, p)=\frac{1}{2}\left[\frac{1}{F(k ; \xi)}+\frac{1}{F(p ; \xi)}\right], \\
& b(k, p)=\frac{1}{2} \frac{1}{\left(k^{2}-p^{2}\right)}\left[\frac{1}{F(k ; \xi)}-\frac{1}{F(p ; \xi)}\right], \\
& c(k, p)=-\frac{1}{\left(k^{2}-p^{2}\right)}\left[\frac{\mathcal{M}(k ; \xi)}{F(k ; \xi)}-\frac{\mathcal{M}(p ; \xi)}{F(p ; \xi)}\right] .
\end{aligned}
$$

The complexity of this vertex ansatz is reflected in the fact that there is no covariant gauge where the wavefunction renormalization becomes trivial as in the case of the rainbow approximation. Solutions far away from the Landau gauge are difficult to construct for this vertex. Nevertheless, if we apply the LKFT strategy to the solution of the SDE with the 


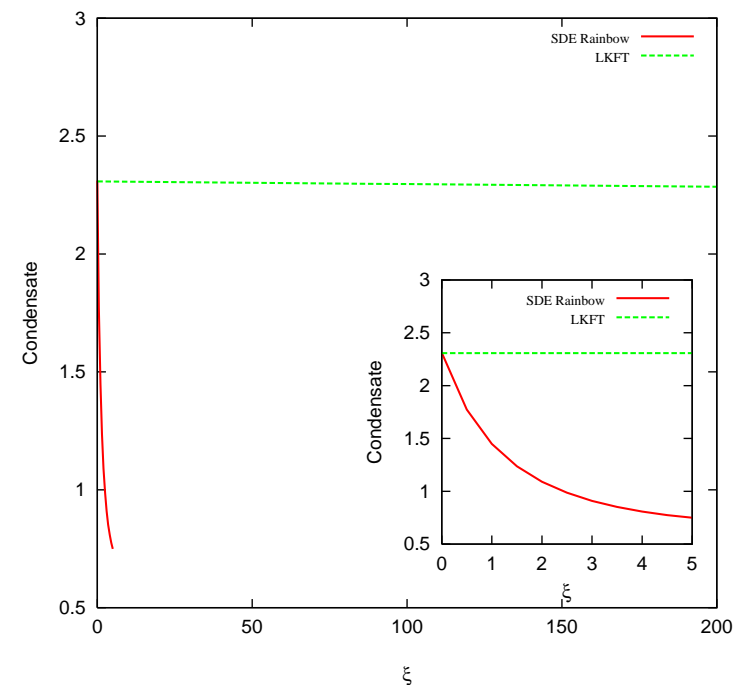

FIG. 4: Gauge dependence of the chiral condensate (in units of $10^{-3} e^{4}$ ) in the Rainbow approximation: LKFT (green-dashed) vs. SDE (red-solid).

BC-vertex in the Landau gauge to other gauges and compute the chiral condensate, we find that this quantity is virtually gauge independent, as can be seen from Fig. 5 (green-dashed line), where a comparison against SDE results (red-solid line) is also shown. Again, a linear fit reveals that the gauge dependence of the condensate is $\langle\bar{\psi} \psi\rangle \simeq 3.21685-0.000157548 \xi$, leading to a vanishing condensate for $\xi_{v}>20000$, a behavior very similar to that of the Rainbow approximation.

\section{B. Unquenched QED3}

In this case the SDE for the fermion propagator, eq. (1) has to be simultaneously solved with the corresponding SDE for the photon propagator with $N_{f}$ flavors of degenerate fermions :

$$
\begin{aligned}
& \Delta_{\mu \nu}^{-1}(q)=\Delta_{\mu v}^{0-1}(q) \\
& -N_{f} e^{2} \int \frac{d^{3} k}{(2 \pi)^{3}} \operatorname{Tr}\left[\gamma_{\mu} S(k ; \xi) \Gamma_{v}(k, q ; \xi) S(q ; \xi)\right] .
\end{aligned}
$$

This exercise has been carried out in [2] employing a hybrid choice of the three point interaction. For the fermion propagator, the well-tested Curtis-Pennington vertex (CP-vertex) [14] was used, whereas the BC-vertex was implemented in the SDE of the photon propagator to avoid unwanted divergences. Unknowns $F, \mathcal{M}$ and $\mathcal{G}$ are found through the equations :

$$
\begin{aligned}
& \frac{1}{F(p ; \xi)}=1+\frac{\alpha}{2 \pi^{2} p^{2}} \int \frac{d^{3} k}{q^{2}} \frac{F(k ; \xi)}{k^{2}+\mathcal{M}^{2}(k ; \xi)}\left[\mathcal { G } ( q ) \left\{\frac{a(k, p)}{2 q^{2}}\left(-q^{4}+\left(k^{2}-p^{2}\right)^{2}\right)-\left[\frac{1}{F(k ; \xi)}-\frac{1}{F(p ; \xi)}\right] \frac{\Omega(k, p)}{2}\left(k^{2}+p^{2}-q^{2}\right)\right.\right. \\
& \left.-\left[\frac{b(k, p)\left(k^{2}+p^{2}\right)-c(k, p) \mathcal{M}(k ; \xi)}{2 q^{2}}\right]\left(-q^{4}+2 q^{2}\left(k^{2}+p^{2}\right)-\left(k^{2}-p^{2}\right)^{2}\right)\right\} \\
& \left.+\xi\left\{\frac{a(k, p)}{2 q^{2}}\left(q^{2}\left(k^{2}+p^{2}\right)-\left(k^{2}-p^{2}\right)^{2}\right)-b(k, p) \frac{\left(k^{2}-p^{2}\right)^{2}}{2 q^{2}}\left(k^{2}+p^{2}-q^{2}\right)+\frac{c(k, p)}{2 q^{2}} \mathcal{M}(k ; \xi)\left(\left(k^{2}-p^{2}\right)^{2}-q^{2}\left(k^{2}-p^{2}\right)\right)\right\}\right], \\
& \frac{\mathcal{M}(p ; \xi)}{F(p ; \xi)}=\frac{\alpha}{2 \pi^{2}} \int \frac{d^{3} k}{q^{2}} \frac{F(k ; \xi)}{k^{2}+\mathcal{M}^{2}(k ; \xi)}\left[\mathcal { G } ( q ) \left\{2 a(k, p) \mathcal{M}(k ; \xi)-\mathcal{M}(k ; \xi)\left[\frac{1}{F(k ; \xi)}-\frac{1}{F(p ; \xi)}\right] \Omega(k, p)\right.\right. \\
& \left.+\left[\frac{2 b(k, p) \mathcal{M}(k ; \xi)+c(k, p)}{2 q^{2}}\right]\left(-q^{4}+2 q^{2}\left(k^{2}+p^{2}\right)-\left(k^{2}-p^{2}\right)^{2}\right)\right\} \\
& \left.+\xi\left\{a(k, p) \mathcal{M}(k ; \xi)+b(k, p) \mathcal{M}(k ; \xi) \frac{\left(k^{2}-p^{2}\right)^{2}}{q^{2}}+\frac{c(k, p)}{2 q^{2}}\left(k^{2}-p^{2}\right)\left(k^{2}-p^{2}-q^{2}\right)\right\}\right], \\
& \frac{1}{\mathcal{G}(q)}=1-\frac{N_{f} \alpha}{2 \pi^{2}} \int d^{3} k \frac{F(k ; \xi)}{k^{2}+\mathcal{M}^{2}(k ; \xi)} \frac{F(q ; \xi)}{q^{2}+\mathcal{M}^{2}(q ; \xi)}\left[a(k, q)\left[W_{1}(k, p)+W_{2}(k, p) \mathcal{M}(k ; \xi) \mathcal{M}(q ; \xi)\right]\right. \\
& \left.+b(k, q)\left[W_{3}(k, p)+W_{4}(k, p) \mathcal{M}(k ; \xi) \mathcal{M}(q ; \xi)\right]-c(k, q)\left[W_{5}(k, p) \mathcal{M}(q ; \xi)+W_{6}(k, p) \mathcal{M}(k ; \xi)\right]\right\}
\end{aligned}
$$

where

$$
\Omega(k, p)=-\frac{k^{4}-p^{4}}{\left(k^{2}-p^{2}\right)^{2}+\left(\mathcal{M}^{2}(p ; \xi)+\mathcal{M}^{2}(k ; \xi)\right)}
$$

is the $\mathrm{CP}$ transverse factor and the $W_{i}(k, p), i=1, \ldots, 6$ are basic functions of momenta given in Ref. [2], 


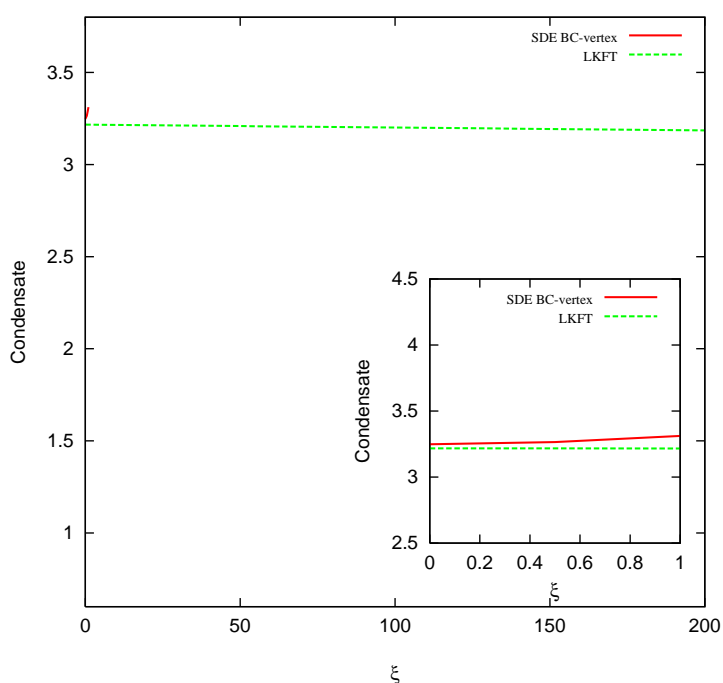

FIG. 5: Gauge dependence of the chiral condensate (in units of $10^{-3} e^{4}$ ) for the $\mathrm{BC}$-vertex in quenched QED3: LKFT (greendashed) vs. SDE (red-solid).

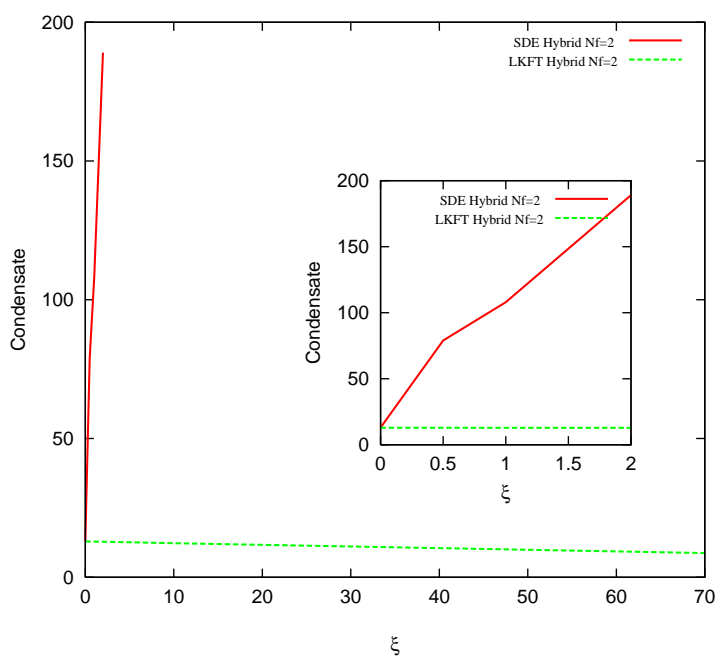

FIG. 6: Gauge dependence of the chiral condensate (in units of $10^{-5} e^{4}$ ) in unquenched QED3 for $N_{f}=2$ in various gauges for the hybrid CP-BC vertex: LKFT (green-dashed line) vs. SDE (red-solid line).

\begin{tabular}{cccccccc}
\hline$\xi$ & $N_{f}=0$ & $N_{f}=1$ & $N_{f}=2$ & $N_{f}=3$ & $N_{f}=4$ & $N_{f}=5$ & $N_{f}=6$ \\
\hline 0 & 333 & 121 & 13 & 0.026 & $? ?$ & 0 & 0 \\
0.5 & 340 & 165 & 79 & 39 & 23 & 15 & 11 \\
1 & 351 & 202 & 108 & 74 & 55 & 37 & 29 \\
2 & 356 & 259 & 189 & 143 & 107 & 92 & 77 \\
\hline
\end{tabular}

TABLE I: $-\langle\bar{\psi} \psi\rangle$ from Ref. [2] in units of $10^{-5} e^{4}$.

This hybrid choice of the vertex made in [2] yields results which show a severe gauge dependence for the condensate.
There are even values of $N_{f}$ for which the dynamical breakdown of chiral symmetry takes place in one gauge and not in another, as can be seen by looking at the entries in Table I we have taken from [2]. The use of different vertex ansätze in the coupled equations gives rise to an internal inconsistency.

But LKFT can help in this case as well. Starting from the solution in the Landau gauge, we can apply the LKFT strategy. In Fig. 6, we plot the condensate value for $N_{f}=2$ as a function of $\xi$ for the two methods. Along the (almost) flat (green-dashed) line, both the WGTI and the LKFT are satisfied (and the inconsistency stemming from the use of the hybrid vertex ansatz is minimized), whereas, in the sharply rising graph (red-solid line), LKFT are violated although the WGTI continues to hold true. One can thus make a gauge independent statement that assuming the BC-CP truncation scheme to be valid in the Landau gauge, the critical number of flavors would lie in the range $3<\left(N_{f}\right)_{c}<5$. Note that for the unquenched QED, transverse vertex in principle knows about the photon propagator, [24]. We suspect that the slight gauge dependence in Fig. 6 stems from the absence of this dependence in the CP-vertex.

\section{DISCUSSION AND CONCLUSIONS}

To conclude, the inclusion of the WGTI alone is not sufficient to ensure the gauge independence of the physical observables associated with the fermion propagator. It is essential to apply LKFT to the dynamically generated mass function as advocated in $[11,12]$. This is what we achieve in an exact numerical fashion. The truncations employed correspond to approximating the fermion-boson interaction by the bare vertex, the $\mathrm{BC}$-vertex and a hybrid choice of the CP-BC vertex. Numerically, we obtain virtually gauge independent value of the condensate for a very broad range of values of the covariant gauge parameter for all the above-mentioned cases.

As discussed in Ref. [11], it is important to note that the LKFT do not generate the soultion in the starting gauge. However, it is not unnatural to start from the Landau gauge solution as the starting one as we do not expect the truncations employed in this work to yield significantly different results in this gauge. Some more definitive conclusions can only be drawn after a detailed study of the LKFT for the vertex function itself. Once we know the result in the Landau gauge, LKFT will guide us along the path of varying gauge. A natural extension of this work is to study the gauge covariance of the quark propagator through the generalized LKFT of QCD. In analogy with the present work we expect gauge independence there too.

AR is grateful for the hospitality of the organizers of the IRQCD in Rio Workshop. AB wishes to acknowledge a short term visitor grant by a joint scheme of The Royal Society, U.K, and The Mexican Academy of Sciences, Mexico. Support has also been provided by CIC and CONACyT (grants 4.10 and 46614-I). 
[1] P. Maris and C.D. Roberts, Phys. Rev. C56, 3369 (1997).

[2] C.S. Fischer, R. Alkofer, T. Dahm, and P. Maris, Phys. Rev. D 70, 073007 (2004)

[3] N. Dorey and N.E. Mavromatos, Nucl. Phys. B386, 614 (1992); K. Farakos and N.E. Mavromatos, Mod. Phys. Lett. A13, 1019 (1998); M. Franz and Z. Tesanovic, Phys. Rev. Lett. 87, 257003 (2001); I.F. Herbut, Phys. Rev. B66, 094504 (2002), M. Franz, Z. Tesanovic, and O. Vafek, Phys. Rev. B 66, 054535 (2002).

[4] M. Sutherland et. al., Phys. Rev. Lett. 94, 147004 (2005).

[5] V.P. Gusynin and S.G. Sharapov, Phys. Rev. Lett. 95, 146801 (2005); K.S. Novoselov et. al., Nature 438, 197 (2005). Y. Zhang et. al, Nature 438201 (2005).

[6] S.J. Hands, J.B. Kogut, L. Scorzato, and C.G. Strouthos, Phys Rev. B 70, 104501 (2004); S.J. Hands, J.B. Kogut, and C.G. Strouthos, Nucl. Phys. B645 321 (2002); S.J. Hands, J.B. Kogut, L. Scorzato, and C.G. Strouthos, Nucl. Phys. Proc. Suppl. 119, 974 (2003); V.P. Gusynin and M. Reenders, Phys. Rev. D68, 025017 (2003); M.R. Pennington and D. Walsh, Phys. Lett. B253, 246 (1991).

[7] T. Appelquist, L.C.R. Wijewardhana, Quantum Theory and Symmetries, Cincinnati, 177, (2003); I. Mitra, R. Ratabole and H.S. Sharatchandra, hep-th/0601058.

[8] J.C. Ward, Phys. Rev. 78, (1950); H.S. Green, Proc. Phys. Soc. (London) A66, 873 (1953); Y. Takahashi, Nuovo Cimento 6, 371 (1957).

[9] N.K. Nielsen, Nucl. Phys. B101, 173 (1975); O. Piguet and K. Sibold, Nucl. Phys. B 253, 517 (1985).

[10] L.D. Landau and I.M. Khalatnikov, Zh. Eksp. Teor. Fiz. 29, 89 (1956); L.D. Landau and I.M. Khalatnikov, Sov. Phys. JETP 2, 69 (1956); E.S. Fradkin, Sov. Phys. JETP 2, 361 (1956); K.
Johnson and B. Zumino, Phys. Rev. Lett. 3351 (1959); B. Zumino, J. Math. Phys. 1, 1 (1960). S. Okubo, Nuovo Cim. 15, 949 (1960). I Bialynicki-Birula. Nuovo Cim. 17, 951 (1960). H. Sonoda, Phys. Lett. B499, 253 (2001).

[11] A. Bashir and A. Raya, hep-ph/0511291.

[12] A. Bashir and A. Raya, Nucl. Phys. B709, 307 (2005); A. Bashir and A. Raya, Nucl. Phys. Proc. Suppl. B141, 259 (2005); A. Bashir and A. Raya, proceedings of "2004 International Workshop on Dynamical Symmetry Breaking”, 257 (2004).

[13] J.S. Ball and T.-W. Chiu, Phys. Rev. D22, 2542 (1980).

[14] D.C. Curtis and M.R. Pennington, Phys. Rev. D42, 4165 (1990).

[15] C.J. Burden and C.D. Roberts, Phys. Rev. D44, 540 (1991).

[16] Z. Dong, H.J. Munczek, and C.D. Roberts, Phys. Lett. B333, 544 (1994).

[17] A. Bashir and M.R. Pennington, Phys. Rev. D50, 7679 (1994); Phys. Rev. D 53, 4694 (1996).

[18] A. Bashir, A. Kızılersü, and M.R. Pennington, Phys. Rev. D 57, 1242 (1998)

[19] A. Bashir, A. Kızılersü, and M.R. Pennington, Phys. Rev. D 62, 085002 (2000).

[20] A. Bashir and A. Raya. Phys. Rev. D64, 105001 (2001).

[21] A. Bashir and A. Raya, in "Trends in Boson Research", edited by A.V. Ling, Nova Science Publishers, Inc. N.Y., (2006).

[22] A. Bashir, A. Huet, and A. Raya. Phys. Rev. D 66, 025029 (2002).

[23] V.A. Miransky, "Dynamical Symmetry Breaking in Quantum Field Theories", World Scientific, (1993), pp. 244-245.

[24] A. Kızılersü, Ph.D. thesis, University of Durham, 1995. 